\title{
High-beta plasma blobs in the morningside plasma sheet
}

\author{
G. Haerendel ${ }^{1}$, W. Baumjohann ${ }^{1}$, E. Georgescu ${ }^{1}$, R. Nakamura ${ }^{1}$, L. M. Kistler ${ }^{2}$, B. Klecker ${ }^{1}$, \\ H. Kucharek ${ }^{1}$, A. Vaivads ${ }^{1}$, T. Mukai ${ }^{3}$, S. Kokubun ${ }^{4}$ \\ ${ }^{1}$ Max-Planck-Institut für extraterrestrische Physik, Garching, Germany \\ ${ }^{2}$ University of New Hampshire, Durham, NH, USA \\ ${ }^{3}$ Institute of Space and Astronautical Science, Sagamihara, Japan \\ ${ }^{4}$ Solar-Terrestrial Environment Lab., Nagoya University, Toyokawa, Japan
}

Received: 28 June 1999 / Revised: 23 July 1999 / Accepted: 17 August 1999

\begin{abstract}
Equator-S frequently encountered, i.e. on $30 \%$ of the orbits between 1 March and 17 April 1998 , strong variations of the magnetic field strength of typically $5-15$-min duration outside about $9 R_{E}$ during the late-night/early-morning hours. Very high-plasma beta values were found, varying between 1 and 10 or more. Close conjunctions between Equator-S and Geotail revealed the spatial structure of these "plasma blobs" and their lifetime. They are typically $5-10^{\circ}$ wide in longitude and have an antisymmetric plasma or magnetic pressure distribution with respect to the equator, while being altogether low-latitude phenomena $\left(\leq 15^{\circ}\right)$. They drift slowly sunward, exchange plasma across the equator and have a lifetime of at least 15-30 min. While their spatial structure may be due to some sort of mirror instability, little is known about the origin of the high-beta plasma. It is speculated that the morningside boundary layer somewhat further tailward may be the source of this plasma. This would be consistent with the preference of the plasma blobs to occur during quiet conditions, although they are also found during substorm periods. The relation to auroral phenomena in the morningside oval is uncertain. The energy deposition may be mostly too weak to generate a visible signature. However, patchy aurora remains a candidate for more disturbed periods.
\end{abstract}

Key words. Magnetospheric physics (plasma convection; plasma sheet; plasma waves and instabilities)

\section{Introduction}

Equator-S had a very short lifetime, and not all of the instruments functioned as planned. The available data set is thus incomplete and restricted to the period 16

Correspondence to: G. Haerendel, e-mail: hae@mpe.mpg.dc
December 1997-30 April 1998, and a local time interval from 11 MLT to 02 MLT. In spite of these rather severe limitations, the mission produced some novel data. This is mainly due to the low inclination $\left(3.9^{\circ}\right)$ and eccentric orbit (apogee $11.5 R_{E}$ geocentric, perigee $500 \mathrm{~km}$ altitude). Not only did this orbit allow the study of the prenoon magnetopause and low-latitude boundary layer, but the spacecraft was also subsequently, i.e. after about 1 March, carried into one of the least explored regions of the outer magnetosphere, the low-latitude plasma sheet between 9 and $11.5 R_{E}$ and 07 and 02 MLT. Because of their higher inclination, most earlier magnetospheric satellites on eccentric orbits missed this region or, when it was encountered, the investigators concentrated on the more spectacular magnetopause crossings. Therefore, little was added to the pioneering work of Heppner et al. (1967) based on the OGO-A fluxgate magnetometer measurements.

OGO-A could explore the local time sector from 04:30 to $06: 30$ at geomagnetic latitudes between $\pm 15^{\circ}$ out to and beyond the magnetopause on a number of inbound passes. The main findings were: (a) a vanishing gradient of the total magnetic field between $11 R_{E}$ and the magnetopause, (b) in a number of cases little contrast between the behavior of magnetospheric and magnetosheath fields, and (c) at times higher fields outside rather than inside the magnetopause. Based on these findings, Heppner et al. (1967) concluded that the low-latitude region between $11 R_{E}$ and magnetopause was characterized by a high value of the plasma beta $\left(\beta=2 \mu_{o} p / B^{2} \geq 1\right)$. Furthermore, on a number of outbound passes at latitudes $>24^{\circ}$ and inside the magnetopause, they found intervals of up to 2-min duration during which the magnetic field changed greatly, and suggested the existence of "some degree of blobbiness" of the plasma and field structure, without being able to characterize these features more accurately. Equator-S data can now throw more light on this region.

It will be subsequently shown that in one third of all orbits, between about 9.3 and $11.5 R_{E}$ and SM latitudes below about $13^{\circ}$, extended periods are found during 
which magnetic-field magnitude and direction change dramatically for intervals of several up to $15 \mathrm{~min}$. In a subset of these events, we were able to determine the plasma pressure and found high-beta values, sometimes exceeding 10 in the field depressions. Following the language of Heppner et al. (1967) we name these depressions "plasma blobs". Their longitudinal dimensions are of order $1 R_{E}$.

Complementary information on these blobs may be derived from the conjugate morningside aurora. As will be shown, the locations of their occurrence were conjugate with the equatorward region of the morningside auroral oval between 03:30 and 08:00 MLT. The most characteristic auroral phenomenon of this region is the patchy aurora, typically appearing about $10-30 \mathrm{~min}$ after substorm onset in the midnight section with typical sizes of 100-200 km Akasofu et al. (1966), but also existent during quieter conditions Oguti (1981). The shapes of the patches are quite irregular, and they drift slowly eastward with speeds of a few $100 \mathrm{~m} / \mathrm{s}$. Oguti (1981) interprets the movements as manifestations of real plasma drifts in the magnetosphere.

Our presentation of plasma blobs will first deal with individual observations and characterize their plasma and field structure. Then we will analyze their spatial scales and motions, which was made possible through a few conjunctions of Equator-S with Geotail. Next we will discuss the occurrence of these events in space and according to magnetic activity. Field-line tracing yields the conjugate sites in the ionosphere and leads to a discussion of the relation with the morningside aurora. Finally, we will speculate on the origin of the plasma blobs.

\section{Instrumentation}

The Equator-S magnetic field instrument is fully described in Fornacon et al. (1999). It consists of two units with a pair of three-axes fluxgate magnetometers each. The sensors of the primary and the redundant units are mounted on two rigid booms, with the main sensor located at the end of the $1.8 \mathrm{-m}$ boom and the other 50 $\mathrm{cm}$ further inboard. The sampling rate is 128 vectors/s in normal mode, when only the outboard magnetometer is used, and 64 vectors/s for dual mode operation. The amplitude resolution is 16 bits, and the ranges are selected automatically in steps of 4 between 256 and $64000 \mathrm{nT}$. The data used in the present study are mainly $128 \mathrm{~Hz}$ data, all sampled with a resolution of about $10 \mathrm{pT}$ in the $256 \mathrm{nT}$ mode.

The Ion Composition Instrument (ICI) measures the 3-dimensional distribution functions of the major ion species in the magnetosphere and magnetosheath over the energy per charge range $20-40000 \mathrm{eV} / \mathrm{e}$ Kistler et al. (1998). It is a combination of a top-hat electrostatic analyzer followed by post-acceleration of $15-18 \mathrm{kV}$, and then a time-of-flight measurement, similar to the CIS1 instrument designed for CLUSTER (Rème et al., 1997) and the TEAMS instrument on FAST (Möbius et al., 1998). It can resolve the major ion species, $\mathrm{H}^{+}, \mathrm{He}^{++}$,
$\mathrm{He}^{+}, \mathrm{O}^{++}$, and $\mathrm{O}^{+}$. In the high-rate mode of the data acquisition, full velocity distributions are obtained every spin period $(1.5 \mathrm{~s})$, in low rate every $6 \mathrm{~s}$. The onboard moments are calculated every spin period.

\section{Nature of the plasma blobs}

A common observation of Equator-S in the morningside plasma sheet outside about $9 R_{E}$ and at SM latitudes below $13^{\circ}$ is that of large semiperiodic fluctuations of magnetic field strength and orientation with durations of several minutes. On about one third of all orbits between 1 March and 17 April, i.e. between 07 MLT and 02 MLT such large-amplitude fluctuations were observed. Figure 1 shows a striking example.

The plasma data obtained with the ICI Kistler et al. (1998) show corresponding variations of the plasma pressure, in antiphase with the magnetic pressure, albeit with much smaller relative amplitude. The long timescales of the variations in the presence of magnetoacoustic speeds of the order of $1000 \mathrm{~km} / \mathrm{s}$ suggest magnetostatic rather than dynamic equilibrium between the high and low (magnetic) pressure regions. The observed flow velocities of a few tens of $\mathrm{km} / \mathrm{s}$ confirm this suggestion. The widely different relative amplitudes of the magnetic and plasma pressure variations can only be reconciled if the plasma beta is larger than unity. A direct derivation of the plasma pressure from the ICI using efficiencies determined from ground calibrations gives densities which are too low to produce the expected pressure equilibrium. The reasons for these discrepancies are still being resolved. To overcome this impediment, the following procedure has been employed.

We assume approximately constant total pressure over an extended time interval, typically $1 \mathrm{~h}$, and try to match the measured magnetic field and plasma pressure values with

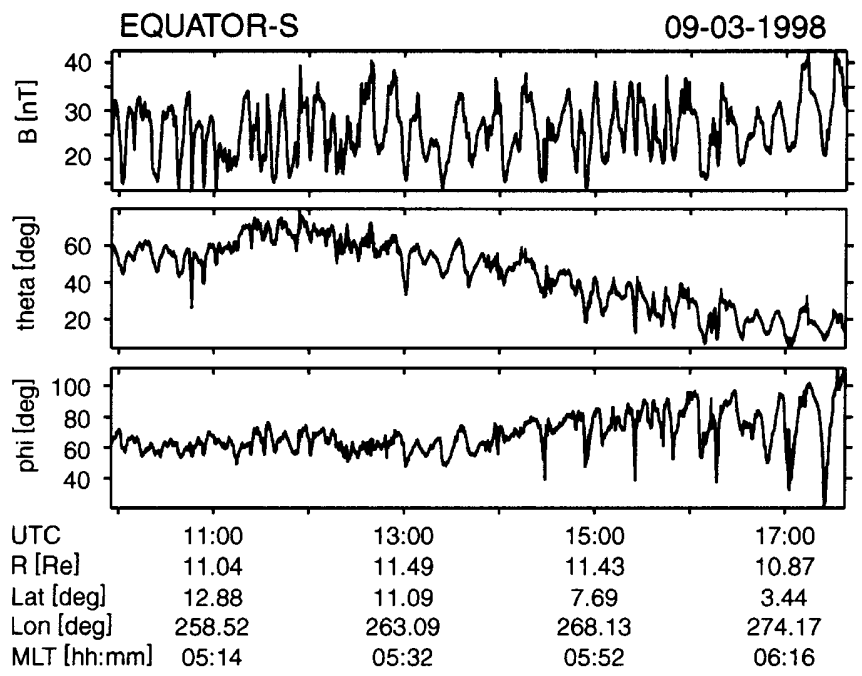

Fig. 1. Large-amplitude fluctuations of magnetic field strength and orientation near the apogee of Equator-S during early morning hours 
$p+\frac{B^{2}}{2 \mu_{o}}=p_{o}+\frac{B_{o}^{2}}{2 \mu_{o}}=$ const.

for a number of individual pressure variations. This means that we disregard the contribution of magnetic shear stresses, which will be justified in retrospect, when we have analyzed the spatial structure of the blobs.

The calibration procedure can be briefly characterized as an attempt to fit the magnetic $\left(p_{B}\right)$, thermal $\left(p_{t h}\right)$ and total pressures $\left(p_{t o t}\right)$ to the equations:

$p_{B}=\alpha p_{t h}+p_{t o t}$.

If our assumption of magnetostatic equilibrium with negligible shear stresses is valid and if $p_{t h}$ were measured reliably, one should find $\alpha=-1$. This is invariably not the case, $-\alpha$ exceeds unity typically by factors of $2-5$. But extrapolation of a linear fit of the scattered data to $p_{t h}=0$ yields $p_{t o t}$. We then correct the measured data of $p_{t h}$ by multiplying them with $-\alpha$.

One can regard the temperature determination from the energy distribution of the protons as much more reliable than the density derivations. Therefore, the correction $(-\alpha)$ should apply to the density values. In the few cases of close conjunction with the Geotail spacecraft this conclusion is supported by a comparison with its density measurements.

Figure 2 shows plasma and magnetic field measurements and derived quantities from an outbound orbit of Equator-S on 18 March, 1998. At about $9 R_{E}$ (14:37 UTC) a change in the character of the magnetic field is observed. It exhibits short-term (i.e. few minutes) variations, a slight drop in magnitude and increasing directional variations. This behavior is obviously connected with an increase in plasma pressure followed by a rather sudden jump shortly before 15:00 UTC. While the spacecraft moves outward and B decreases, the plasma beta grows well above unity. Outside about $10 R_{E}$, beta

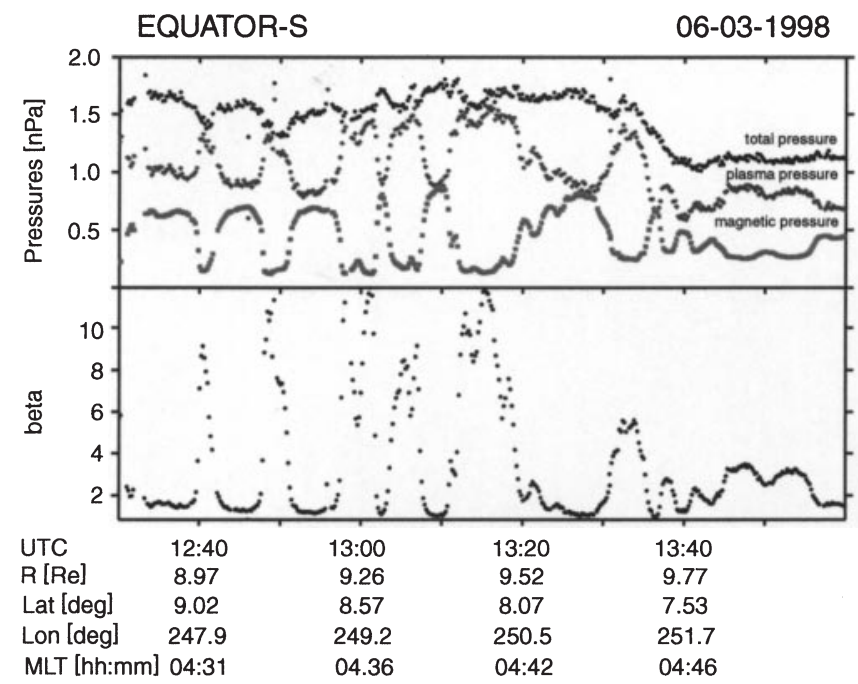

Fig. 2. Plasma and magnetic-field measurements plus derived quantities on an outbound orbit of Equator-S on 18 March 1998. Outside about $10 R_{E}$, large-amplitude variations of magnetic field strength and orientation are found in a high-beta environment undergoes strong variations, which become quite regular between 18 and 19 UTC. It is this period that we used to fix the correction factor $-\alpha(=4.1)$ by postulating constant total pressure. Applying this factor throughout the whole data period, we find a remarkable constancy of the pressure outside about $9 R_{E}$.

Figures 3 and 4 present further examples of strong variations of the magnetic and plasma pressures. Densities and temperatures are in the same range as in Fig. 2. However, not always can one fit the data of a long period with constant total pressure. Temporal changes as seen in Fig. 3 after 13:33 UTC are not uncommon. But nearly constant total pressure for 1 or $2 \mathrm{~h}$ is normally a non-conflicting assumption.

All examples shown demonstrate that the strong variations of magnetic pressure are embedded in a very high-beta environment. Even in the maxima of $B$, beta hardly ever falls below unity, whereas in the minima beta reaches or even exceeds 10 . This finding led to the choice of the name plasma blobs. Obviously, a high-beta plasma (on average $\beta \cong 3$ ) is injected into the morning-
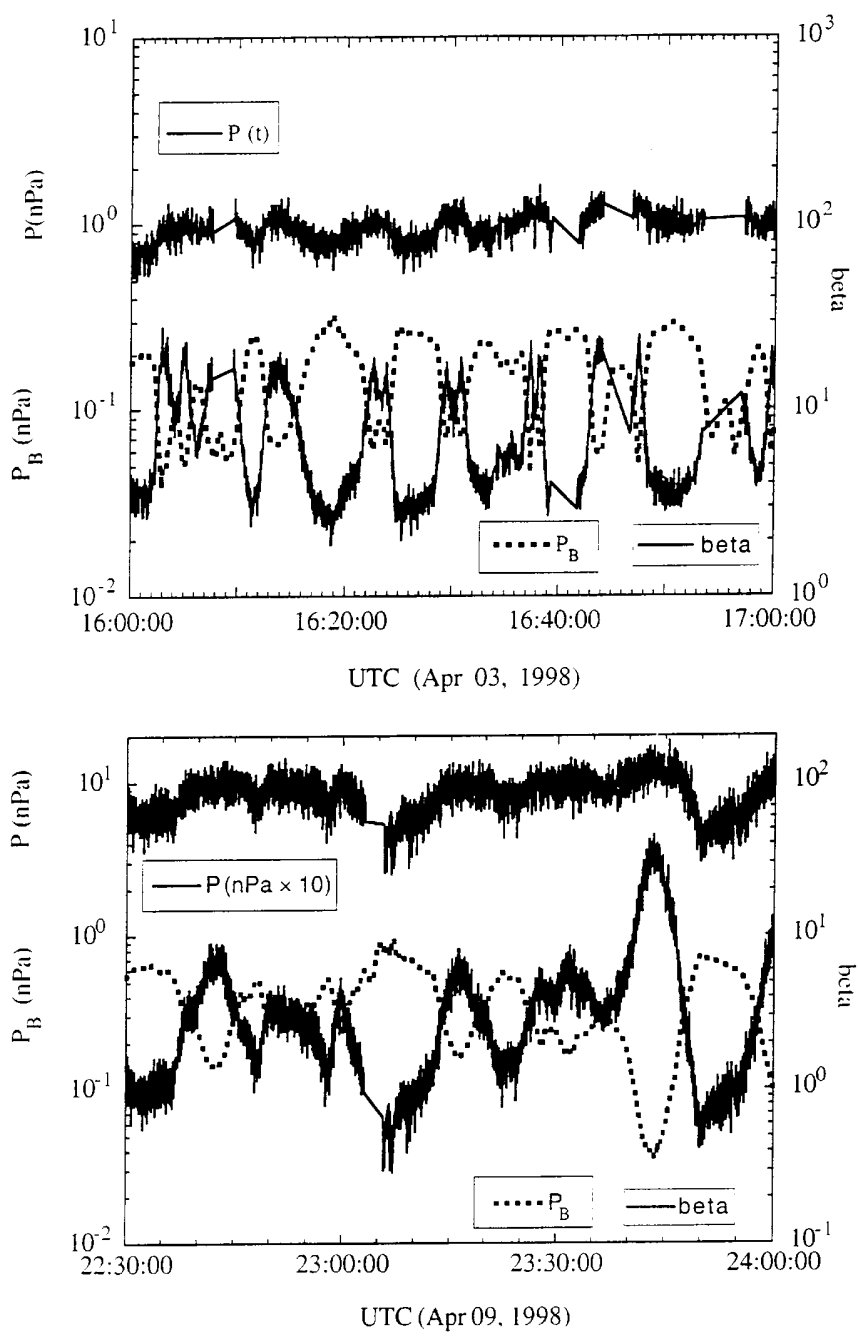

Fig. 3. Recalibration of measured plasma pressure so as to yield approximately constant total pressure for $1 \mathrm{~h}$ (see text) and corresponding plasma beta $\left(2 \mu_{o} p / B^{2}\right)$ on 9 March 1998. After 13:34 UTC, there is a drop in total pressure 

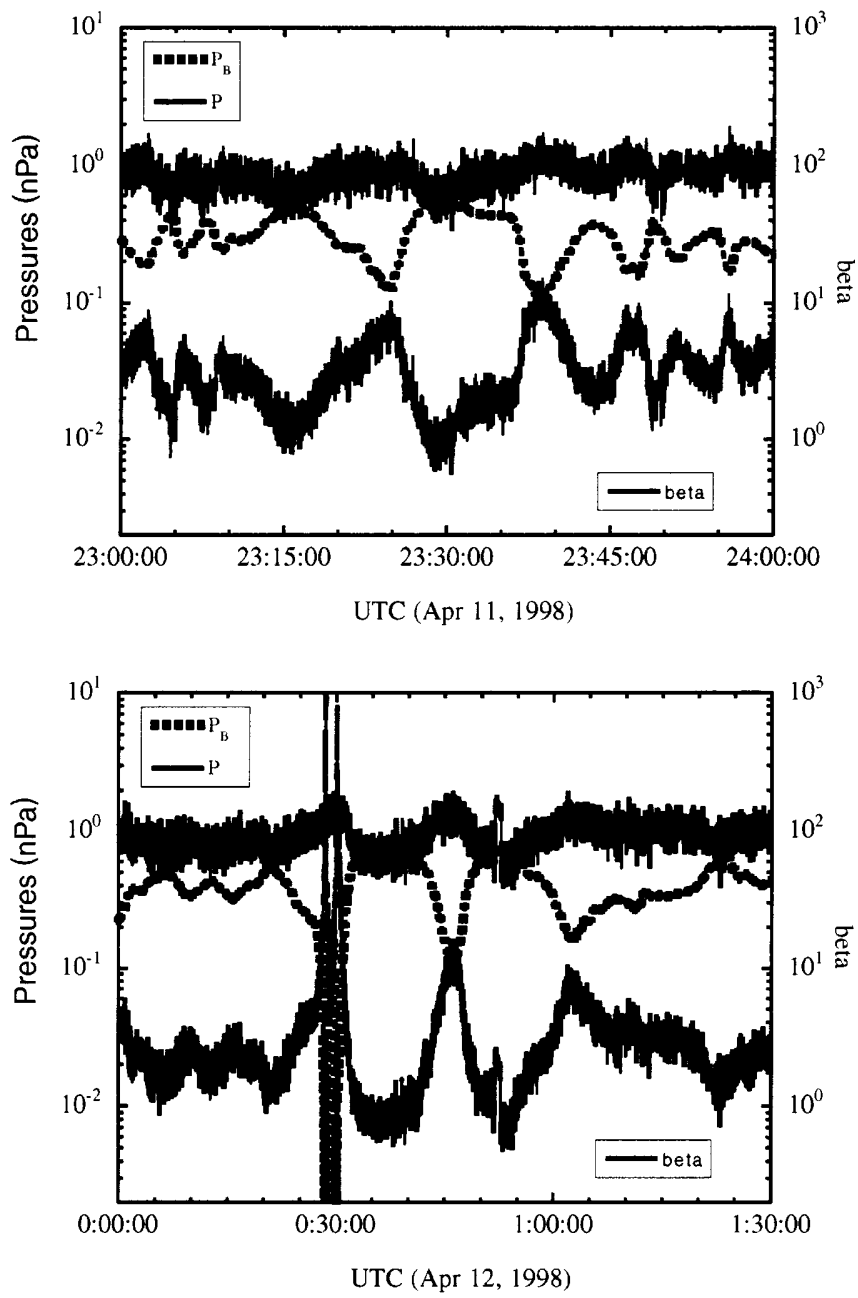

Fig. 4. Same as Fig. 2, but without total pressure, for 3 April and 9 April 1998

side plasma sheet at low latitudes and arranges itself spatially in a way that regions of very high $\beta(\sim 10)$ alternate with a region of $\beta \approx 1$. The easiest way to establish such a configuration is by redistribution of the hot plasma along magnetic field lines. We will return to this point after having analyzed the spatial structure.

Similar high-beta plasma variations had already been observed by Geotail in the early morning sector, a few $R_{E}$ further outward than reported here and have been interpreted as slow mode or mirror mode waves Nishida et al. (1997).

\section{Spatial structure}

The magnetic structure of the plasma blobs can be divided in two classes, regular and irregular. The data of 18 March shown in Fig. 2 are an example of the first class. Figure 5 presents an example of the irregular class. The latter has not yet been subjected to the pressure balance analysis described in the previous section, however, rough inspection confirms also here the highbeta nature. The regular variations are the rarer class. They were observed on about $40 \%$ of the periods with

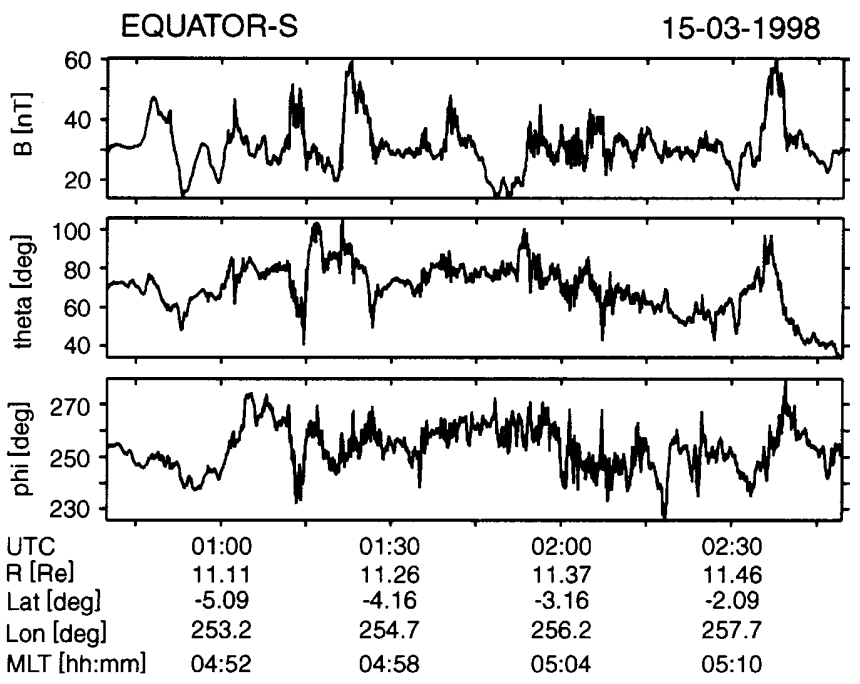

Fig. 5. Large-amplitude irregular variations of the magnetic field (15 March 1998)

strong magnetic variations, mostly for only part of these periods.

Typically the drop in magnetic field strength is accompanied by a characteristic change in field direction in the following sense. With decreasing $B$ also the polar angle, $\Theta$, decreases, i.e. the magnetic vector steepens with respect to the (SM) equatorial plane. The azimuthal angle, $\phi$, changes little, while $B$ is decreasing, but then, in the bottom of the magnetic depression, it changes significantly. Figure 6 shows one out of many examples from the 9 March period (cf Fig. 1). Figure 7 summarizes in a sketch the most commonly observed variations of $B, \Theta, \phi$, within the regular class. The polar angle, $\Theta$, drops typically by about $20^{\circ}$ inside the field depression (plasma blob). The change of azimuth (depending on the value of $\Theta$ ) is mostly, but not always negative, i.e. the field vector is tilted by typically $20^{\circ}$ sunward.

The change in polar angle, as $B$ is dropping and the plasma pressure is increasing, must be related to an inhomogeneous accumulation of the plasma. There are two possibilities. In principle, dense plasma could be supplied from lower altitudes and, while inflating the field, steepen the magnetic vector. The other possibility is that plasma flows in from high altitudes, i.e. lower latitudes or from the opposite hemisphere and assembles non-symmetrically with respect to the equator. The question can be answered by analyzing the parallel component of the plasma flow. For demonstration, we choose data from Geotail for one of the conjunctions with Equator-S. On 9 March the closest approach of GTL and EQS occurred at 12:15 UTC. EQS was at $R=11.4 R_{E}$ and $12.1^{\circ}$ northern SM latitude, whereas GTL was south of the equatorial plane, at a latitude of $-5.8^{\circ}$ and slightly further inward, i.e. at $\mathrm{R}=10.97 R_{E}$. Figure 8 shows, for a $1 \mathrm{~h}$ period around the time of conjunction the mostly field-aligned z-component of the plasma flow and the magnitude of $B$ as measured by Geotail. (The actually measured $v_{z}$ has been shifted by $\sim 20 \mathrm{~km} / \mathrm{s}$, so as to yield an almost vanishing mean velocity. Such a shift of the zero-line is well within the 


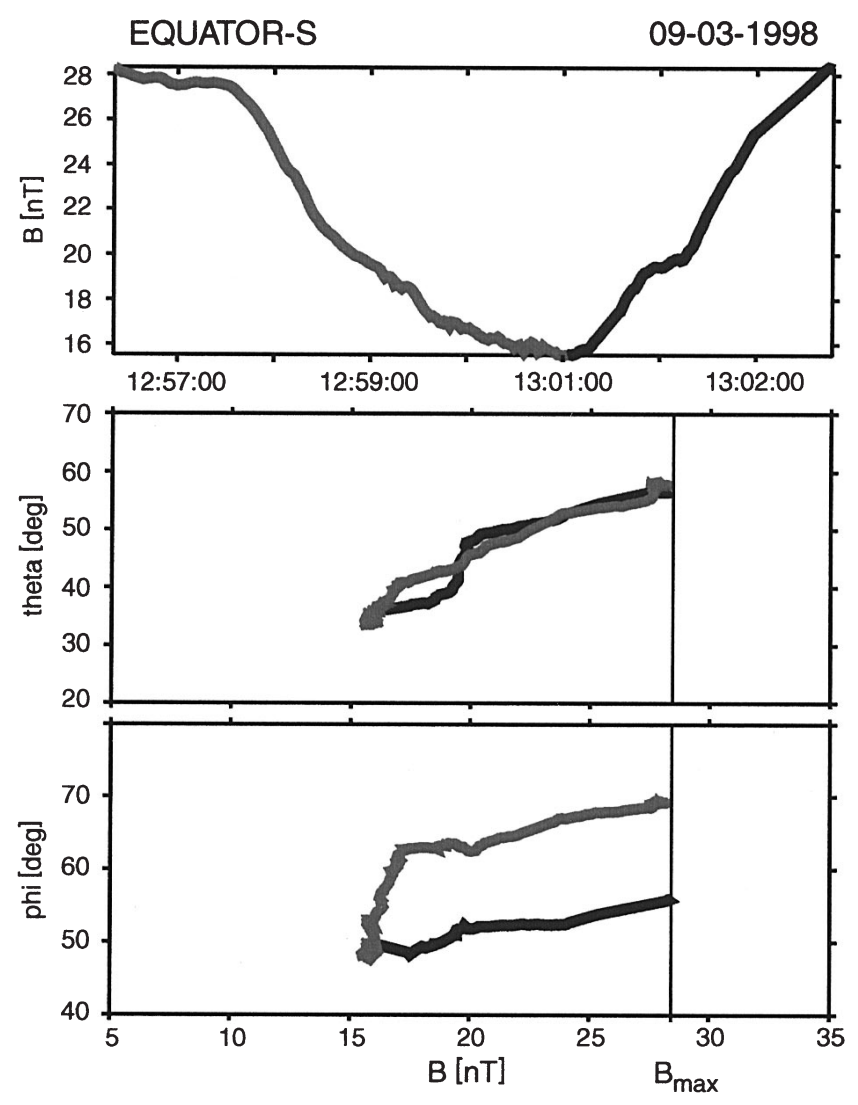

Fig. 6. An example of the detailed variation of magnitude $(B)$, polar angle (theta), and azimuth (phi) of the magnetic field in one of the field depressions (plasma blobs) observed on 9 March 1998. Theta and phi are plotted versus $B$, decreasing phase indicated by thick line

errors of the velocity determination.) With a phase shift of about $90^{\circ} v_{z}$ maximizes before the maximum of $B$. This also holds for the times before and after the interval shown. Because of the anticorrelation of $p$ and $B$, this means that the flow is towards the equator $\left(v_{z}>0\right)$ when $p$ decreases and away from the equator as $p$ is building up. The velocity amplitude ranges between about 20 and $40 \mathrm{~km} / \mathrm{s}$. The transverse velocity components are too small to be reliably interpreted.

These findings imply that the mass reservoir must be at lower latitudes or beyond, in the opposite hemisphere. This is consistent with Heppner et al. (1967) who found the high-beta region to lie below about $15^{\circ}$ latitude. At the position of the spacecraft, plasma is arriving from the equator, the field is inflated and the elevation angle steepens at the low-latitude side of the blobs.

Figure 9 provides a plausible interpretation of plasma movements, field inflation and elevation increase. But it goes a step further by implying a N-S antisymmetry. The existence of such an antisymmetry is revealed by simultaneous measurements north and south of the magnetic equator along essentially the same flux-tube, which were possible during the conjunctions of EQS and GTL on 9 March and 25 April. Figure 10 shows $B$ for both, EQS and GTL, of 9 March for $4 \mathrm{~h}$ around the time of conjunction. Within \pm 15 min of 12:15 UTC (closest approach of EQS and GTL in longitude), the

\section{Typical Magnetic Field Signature of a Plasma Blob}

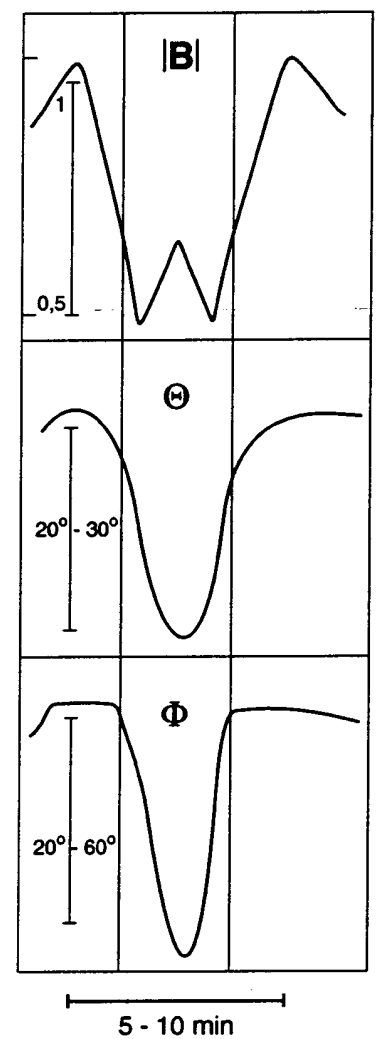

Fig. 7. Summary sketch of typical variations of $B$, theta and phi within a plasma blob

strong variations of $B$ at both spacecraft are clearly in antiphase. Taking $B$ as a proxi for $p$ we find a pressure maximum at EQS when the magnetic pressure has a maximum at GTL and vice versa. Figure 11 is an attempt to visualize this antisymmetric field-plasma distribution.

The antisymmetry of $B$ and $p$ with respect to the equator and the observed flow directions suggest that the blobs owe their existence to a rearrangement of the high-beta plasma alternatingly northward and southward of the central plasma sheet. Build-up of magnetic pressures on one side seems to accelerate the plasma towards the other side of the equator, where it piles up under inflation of the magnetic field.

What is then the reason for the observed quasiperiods of $B$ and $p$ ? Do they show growth and decay of the blobs or rather a convected spatial structure? The conjunctions of EQS and GTL provide the answer. Looking again at Fig. 10, one notices that the variations of $B$ on both spacecraft change from being in antiphase at the time of (longitudinal) conjunction to being in phase at about $1 \mathrm{~h}$ before and after. At these times, EQS and GTL were separated by about $4.5^{\circ}$ in SM longitude. At the equator this corresponds to a separation of 0.85 $R_{E}$ or $\sim 5500 \mathrm{~km}$. Because of the N-S antisymmetry this distance must indicate the half width of an individual blob. The same evaluation for the 25 April conjunction 


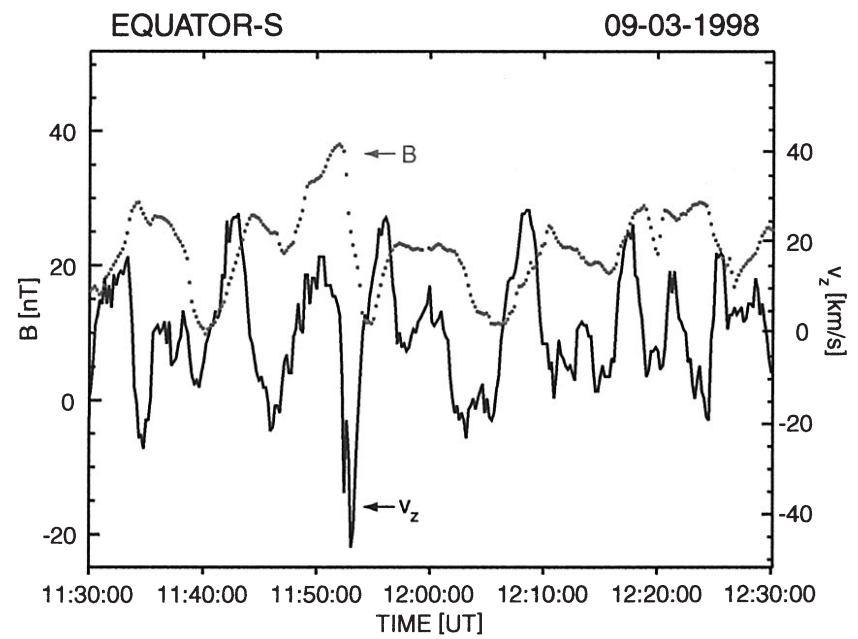

Fig. 8. Phase relation between magnetic field magnitude (dotted line) and field-parallel flow component (full line) as observed by Geotail during the conjunction with Equator-S on 9 March 1999. $v_{z}$ is directed equatorward when $B$ is rising, i.e. when the plasma pressure south of the equator is decreasing and vice versa
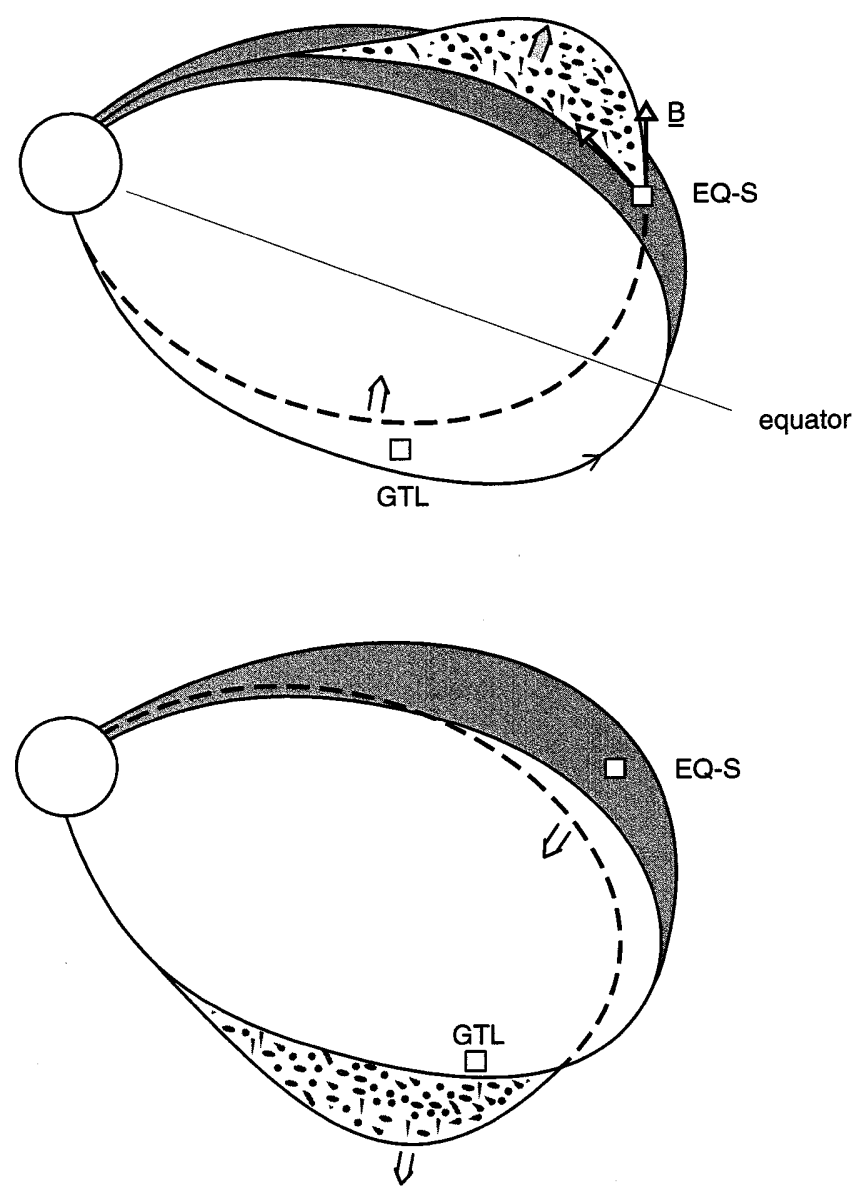

Fig. 9. A view of the field distortions in the meridional plane due to the concentration of plasma and accompanying field inflation at offequatorial latitudes

yields about $8000 \mathrm{~km}$. If we take as typical width $\sim 10000 \mathrm{~km}$ and take into account that EQS or GTL see about seven blobs per $1 \mathrm{~h}$ on 9 March, one derives
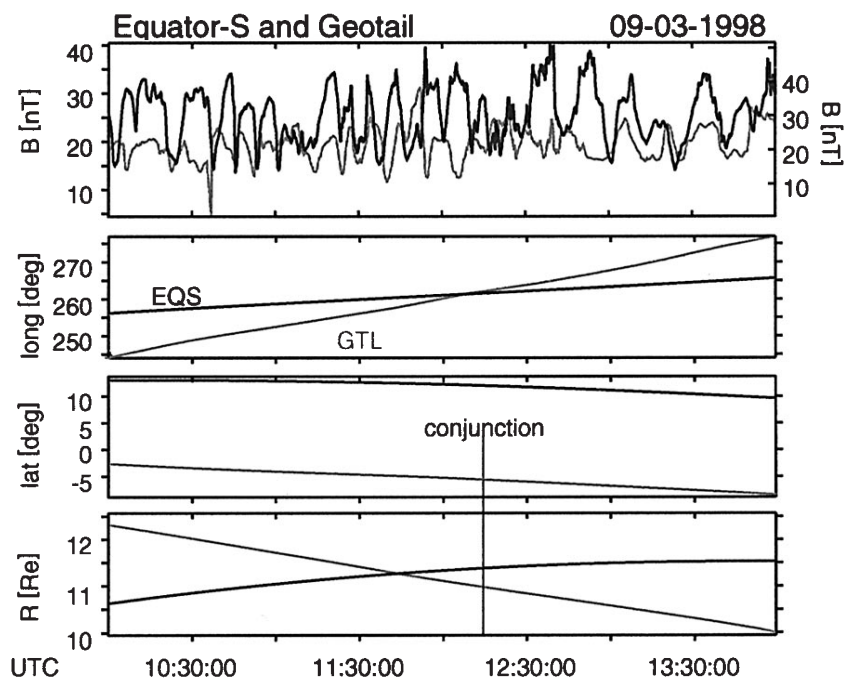

Fig. 10. Phase relation between the magnetic field magnitudes at EQS (thin line) and GTL (thick line) and s/c positions in SM coordinates near the conjunction on 9 March 1998

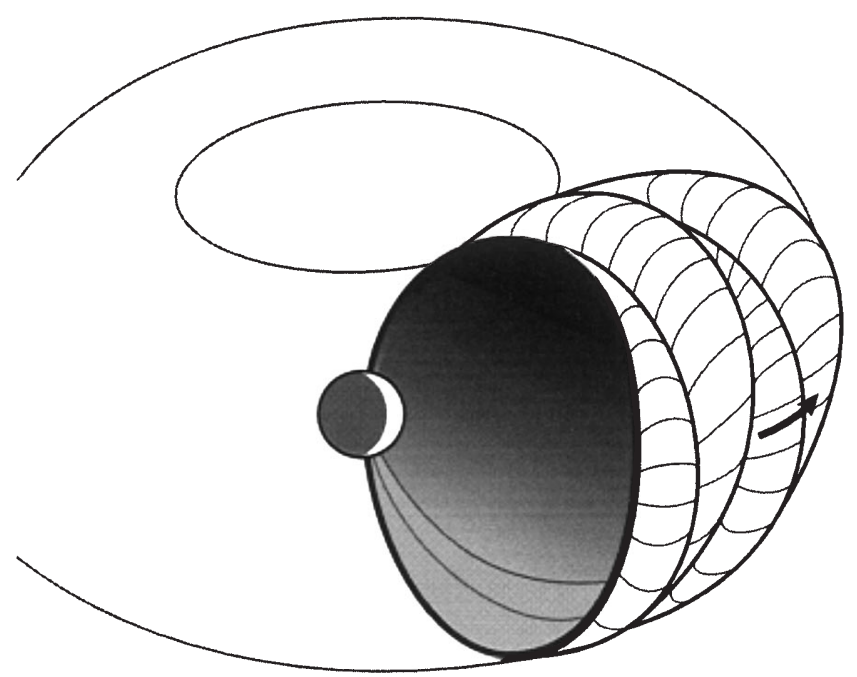

Fig. 11. Sketch of the antisymmetric packing of field inflation and compression caused by the high-beta plasma blobs. The longitudinal separation at $\sim 11 R_{E}$ is of order $1-2 R_{E}$

an average transport velocity of $\sim 20 \mathrm{~km} / \mathrm{s}$ with respect to the spacecraft $\left(v_{G T L} \sim 2.8 \mathrm{~km} / \mathrm{s}\right)$. This is quite consistent with the average $v_{x}$-component (essentially perpendicular to $B$ ) measured with GTL within the uncertainty of about $20 \%$ with which such low velocities can be measured. The positive value of $\bar{v}_{x}$ means sunward transport, quite in agreement with the general direction of plasma convection in the morning sector.

The consistency of the above determination of the transport velocity from width and passage time with the local flow measurements testifies that the blobs have a lifetime substantially longer than the passage time past the satellite. Therefore, if we want to assess the implications of the observed field-aligned flow components of $20-40 \mathrm{~km} / \mathrm{s}$, we must consider transport times well beyond the passage time of $2-4$ min for the rising or 
falling part of a typical blob. We should expect that build-up of a blob requires the displacement of the plasma by a distance exceeding that of Equator-S from the equator, since an asymmetric plasma pressure distribution with respect to the equator has to be established. As the typical steepening of the field elevation angle is found up to SM latitudes of $10^{\circ}$ and beyond, an average field-parallel plasma displacement of $3 R_{E}$ should represent a lower limit. Dividing this length by the above parallel velocity range yields build-up times between 500 and $1000 \mathrm{~s}$, which would correspond to lifetimes at least twice as long, i.e. 17-35 min, and substantially longer than the passage time. An upper limit of the lifetimes cannot be easily established. It would need tracing of individual blobs.

In summary, we have identified the high-beta plasma blobs in the morningside plasma sheet as being created by rearranging dense plasma in a semi-periodic and antisymmetric way (with respect to the equator). Lifetimes are long, substantially longer than the magnetic variations of order 5-10 min seen by the satellites. To call these variations Pc5 is thus not appropriate. They are certainly no field-line resonances or compressional oscillations. The identification as mirror mode waves inside the magnetosphere Nishida et al. (1997) appears to be more appropriate. The slow sunward drift suggests that the ultimate origin of the high-beta plasma is the tail plasma sheet in the late night sector.

With the thus achieved insights into the spatial structure of the plasma blobs, we can now justify the neglect of the magnetic shear stresses in Eqs. (1) and (2). Inspection of Figs. 1-6 and 10 shows that the magnetic field strength mostly drops rapidly, typically within $1 \mathrm{~min}$, and varies more slowly around $B_{\min }$. With a convection speed of about $20 \mathrm{~km} / \mathrm{s}$ this amounts to a gradient length of the plasma pressure of the order of $1000 \mathrm{~km}$. Even where the field is locally inflated, its curvature radius is still of the order of several $R_{E}$. The pressure balance is therefore essentially maintained by the magnetic normal pressure.

\section{Occurrence}

Large-amplitude magnetic field variations are a common phenomenon in the morningside plasma sheet. EQS encountered such variations on $30 \%$ of the orbits between 1 March and 17 April 1998. Figure 12 shows their occurrence in a SM latitude versus geocentric distance diagram. They are clearly a feature of the outer magnetosphere, outside $\sim 9 R_{E}$ and restricted to low latitudes $\left(\leq 15^{\circ}\right)$. The latter statement is based on the observations of OGO-A Heppner et al. (1967), since the EQS orbit is confined to low latitudes. Also based on the OGO-A and Geotail Nishida et al. (1997) findings we conclude that these fluctuations exist outside the EQS apogee of $11.5 R_{E}$, out to the magnetopause/ boundary layer. At the present state of data reduction, we can, however, not be certain that all of the largeamplitude magnetic fluctuations, regular and irregular, occur in a very high-beta plasma, but it is a natural

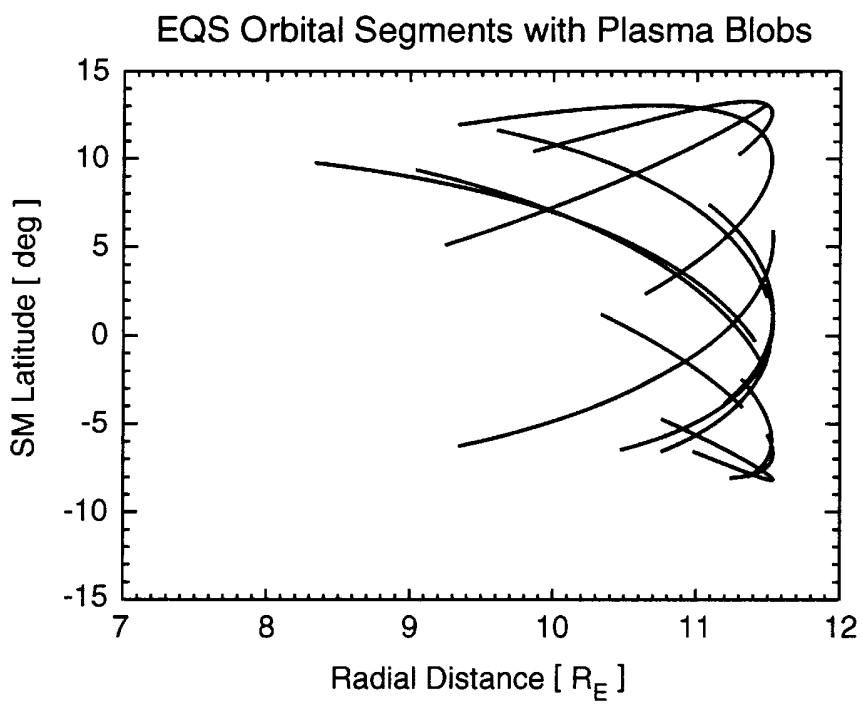

Fig. 12. Segments of the EQS orbit in SM coordinates during which plasma blobs were observed

expectation. This is why we will also use the designation plasma blobs for the periods of large-amplitude magnetic variations, without having the actual evidence from the plasma measurements in each individual case.

A very revealing aspect is the geomagnetic activity at the times of blob encounter. Of 48 data periods of varying length, 13 took place during $\mathrm{Kp}=1$ conditions, 22 during $\mathrm{Kp}=2,12$ during $\mathrm{Kp}=3$, and 1 during $\mathrm{Kp}=4$. The blob occurrence clearly appears to favor geomagnetic quiet conditions, as the distribution over $\mathrm{Kp}=1$ to 4 was 6:7:3:1. Of the class of regular variations, 7 in total, 5 occurred during $\mathrm{Kp}=1$ and 2 during $\mathrm{Kp}=2$.

It is rather surprising that the intrusion of very highbeta plasma and its quasi-periodic spatial organization in the outer morningside magnetosphere should be most efficient during quiet magnetic conditions. That this plasma was a residue of an earlier injection during disturbed, e.g. substorm, conditions, is rather unlikely in view of the observed sunward flow with a speed of typically $20 \mathrm{~km} / \mathrm{s}$. A convection through the midnightto-dawn sector at $11 R_{E}$ would take only $1.5 \mathrm{~h}$. By contrast some of the most pronounced blob periods, e.g. 1 April 1998, were encountered after 4-8 h of extremely quiet magnetic conditions. Therefore, one is led to conclude that it is the preservation of the high-beta nature and of the blobby structure that determines the occurrence rate. During disturbed periods, the magnetization of $\beta \gg 1$ plasma may just proceed more rapidly.

\section{Relation to the aurora}

The role of the plasma blobs as a potential source region of the aurora is an interesting question. The high-beta plasma inside the magnetosphere does not only inflate the magnetic field, but it appears to be convected sunward against the friction exerted by the ionosphere on the corresponding flux-tubes. So, field-aligned currents are 
generated, and auroral phenomena may be associated. Figure 13 exhibits the traces of the orbital segments with blob occurrence (see Fig. 12) in the conjugate northern ionosphere, superposed on the location of the statistical auroral oval of Feldstein (1963). The tracing was performed by using the 1989 Tsyganenko model (Tsyganenko, 1989) for the actual values of Kp. Since the field inflation due to presence of the high-beta plasma at low latitudes is not incorporated in the Tsyganenko model, a correction of the conjugate latitude by about $1^{\circ}$ or so, may be appropriate, but was not applied. Figure 13 thus seems to suggest a concentration of the blobs to the equatorward side of the auroral oval, but one has to remember the $11.5 R_{E}$ apogee of EQS which did not allow to explore field lines with conjugate magnetic latitudes beyond $\sim 71^{\circ}$. If, as suspected above and confirmed by the Geotail measurements of Nishida et al. (1997), the high-beta plasma with blobby structure exists out to the magnetopause, they should have conjugate traces all over the morningside oval.

Projection of longitudinal extent and the transport velocity of the plasma blobs to the conjugate ionosphere yields $\mathrm{E}-\mathrm{W}$ scales of $\sim 200 \mathrm{~km}$ and drift speeds along the oval of $\sim 400 \mathrm{~m} / \mathrm{s}$. These numbers as well as the occurrence in the morning sector are reminiscent of the patchy aurora found in the auroral oval near dawn Akasofu et al. (1966). However, there are crucial differences. The patchy aurora typically appears some 15-30 min after the onset of a substorm in the midnight sector, it develops out of a homogeneous arc at the

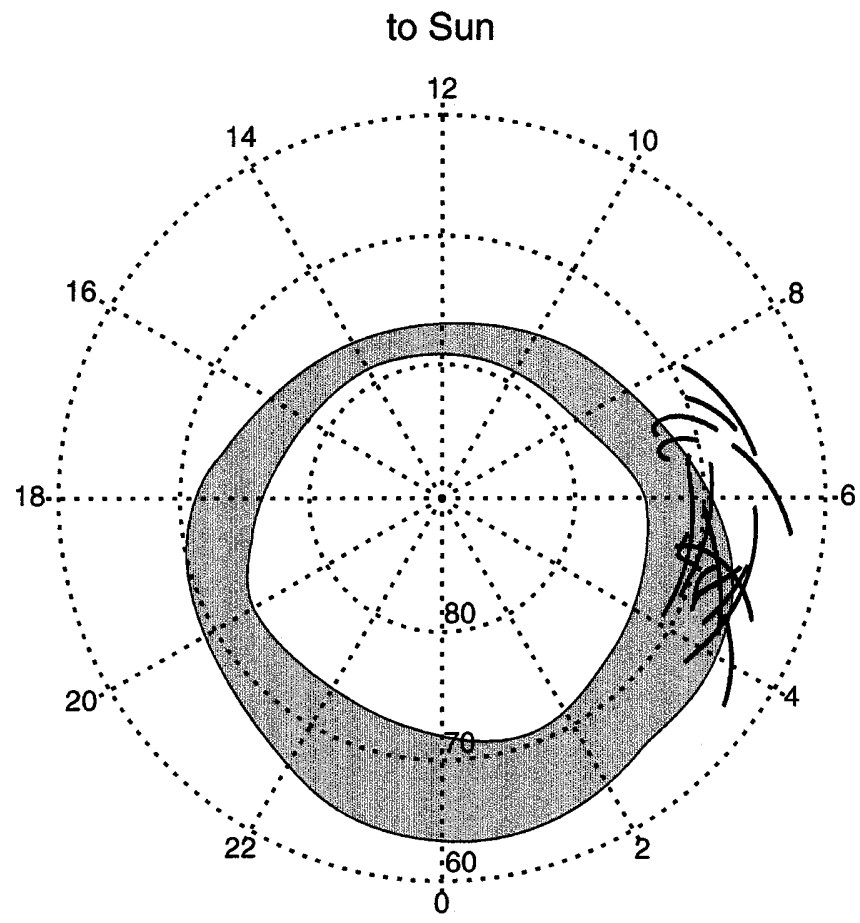

\section{ConjugateTraces of Plasma Blob Segments}

Fig. 13. Traces of the orbital segments shown in Fig. 13 in the northern polar ionosphere superposed on the statistical auroral oval of Feldstein (1963) equatorward edge of the oval and is hardly ever observed poleward of dip latitudes of $70^{\circ}$. The plasma blobs, on the other hand, do not seem to be restricted to the inner edge of the plasma sheet, more importantly, they favor quiet conditions. They can even exist during long periods of $\mathrm{Kp}=1$, whereas in the morningside oval "quiet and homogeneous arcs are common auroral forms" Akasofu et al. (1966).

On the other hand, the occurrence of aurora in the dawn sector is not restricted to substorm recovery. Oguti (1981) describes the existence of patchy aurora poleward of $70^{\circ}$ at dawn during $0 \leq K p \leq 1$. Davis (1963) found a high occurrence rate $(\geq 67 \%)$ at Byrd, Antarctica $(-70.6$ geomagn. lat.) until about 05 local time, which drops drastically during subsequent hours. The aurora exhibits rayed arcs on the southern (i.e. poleward) side, diffuse homogeneous arcs further north, and equatorward thereof diffuse surfaces. Eastward motions prevail. Often these motions are seen to cease while the aurora is still observable for several tens of minutes. Sometimes a westward reversal occurs during late-night hours.

While a detailed auroral counterpart of the plasma blobs can not be identified at this stage of our investigation, we can conclude that there are observable auroral signatures in the conjugate ionosphere, but they seem to fade rapidly at local times beyond about 05 U.T. This implies that the energy content of the blobs is decreasing during these hours until they do no longer give rise to auroral particle acceleration. A quick estimate confirms this suspicion.

A projected drift speed of $400 \mathrm{~m} / \mathrm{s}$ corresponds to an essentially southward pointing electric field of $20 \mathrm{mV} / \mathrm{m}$ in the auroral ionosphere. Even at quiet times at sunrise, there is a high Pedersen conductivity (up to $8 \mathrm{mho}$ ) created by diffuse aurora, but it falls off rapidly with increasing latitude Newell et al. (1996). $\Sigma_{p} \cong 6$ mho may be a good assumption, not more than probably an upper limit. So, underneath the blobs an integrated Pedersen current, $J_{p}$, of $0.12 \mathrm{~A} / \mathrm{m}$ may flow. If the $\mathrm{N}-\mathrm{S}$ scale, $\ell_{N}$, of the blobs is not dissimilar to the $\mathrm{E}-\mathrm{W}$ scale, this current should connect to a field-aligned current of $J_{p} /\left(0.5 \cdot \ell_{n}\right)$, which is at best $1.2 \mu \mathrm{A} / \mathrm{m}^{2}$, if $\ell_{N}$ is only $\sim 200 \mathrm{~km}$. Such field-aligned current strengths are only marginally sufficient to set up an auroral acceleration region. Without post-acceleration, the associated flux of plasma sheet electrons, whose temperature should be of order $T_{p} / 7$, i.e. $\sim 400-500 \mathrm{eV}$, would be close to $0.6 \mathrm{ergs} /$ $\mathrm{cm}^{2} \mathrm{~s}$, too low for a visual signature. However, plasma blobs of higher energy content may be the magnetospheric origin of patchy aurora during the recovery phase of a substorm, or of the rayed aurora typical for dawn during quieter hours [Hallinan, 1999; private communication].

\section{Summary and discussion}

We have presented data from the Equator-S mission, supplemented by measurements of the Geotail satellite, showing the existence of very high-beta plasma blobs as a common phenomenon in the morningside plasma 
sheet. The plasma beta ranges from about 1 to 10 and more. The plasma and magnetic pressure distributions are antisymmetric with respect to the magnetic equatorial plane, their $\mathrm{E}-\mathrm{W}$ extent is $1-2 R_{E}$ and their lifetime at least 1000-2000 s. They drift slowly east- and sunward at speeds of typically $20 \mathrm{~km} / \mathrm{s}$. They have a preference for magnetically quiet periods, in particular the more regular variations, but can also exist during substorm conditions. Their overall occurrence rate is near $30 \%$. One additional and perhaps surprising feature is that in spite of the large variations in magnetic field strength (and beta) the orientation of the field is still reminiscent of a dipole geometry and, far from being chaotic, exhibits very systematic deviations from the model fields which, of course, do not incorporate the existence of such blobs.

There are essentially two questions raised by the discovery of these blobs. What is their nature and what their origin? Taking the high beta for a moment for granted, we should first concentrate on their lifetime and the N-S antisymmetry. The lifetime of $15-30 \mathrm{~min}$ minimum precludes the interpretation as Pc5-type field-line resonances Jacobs (1970) coupled to or excited by compressional waves, since the travel times of Alfvén waves between the two conjugate hemispheres, which constitute an upper limit to the periods, are at best 5 min. For instance, the Alfvén speed in the high pressure, low field part of a blob $\left(B_{\min } \approx 12 \mathrm{nT}\right.$, $n_{\max } \simeq 2 \mathrm{~cm}^{-3}$ ) is about $200 \mathrm{~km} / \mathrm{s}$. A field-aligned extent of the pressure maximum of $6 R_{E}$ is certainly an upper limit. An Alfvén wave would need about $3 \mathrm{~min}$ to traverse this part of the field line, while the higher latitudes need no more than additional $2 \mathrm{~min}$. The considerably longer lifetime found testifies that we are dealing here with long-lived plasma intrusions reminiscent of the mirror mode waves found, also by Equator$\mathrm{S}$, in the magnetosheath adjacent to the magnetopause (e.g. Tsurutani et al., 1982; Lucek et al., 1999).

In earlier studies of Pc5 waves encountered at or near geostationary orbit, e.g. Brown et al. (1968); Lanzerotti et al. (1969); Takahashi et al. (1985), and further outward Hedgecock (1976); Yumoto et al. (1983); Greenstadt et al. (1986); Takahashi et al. (1987), the authors attributed the oscillations either to field line resonances (Alfvén waves) or compressional magnetosonic waves excited either by the Kelvin-Helmholtz instability or by the drift mirror instability. Having excluded field line resonances on the grounds of the long lifetimes derived in this study, there remains the possibility of nonoscillatory mirror waves Price et al. (1986) excited by an internal plasma instability, like the drift mirror instability of Hasegawa (1969) or ballooning-mirror instability Cheng and Qian (1994). Because of lack of full information on all dominant plasma constituents and their anisotropies, we can not check whether the conditions for this instability are fulfilled. However, the structuring in longitude and the antisymmetric distribution in latitude may well be the result of the high-beta plasma finding a lower energy state. Indeed, the antisymmetry implies that several degrees off the magnetic equator the flux-tubes are ordered in a way that "belly" and "neck" are alternating, similar to the findings from simulations by Price et al. (1986; their Fig. 8a). This is intuitively the most "economic" packing of inflatable flux-tubes in a dipole geometry. Also Southwood (1976) found for such a geometry instability only for antisymmetric perturbations, albeit for the Alfvén mode and $\beta \ll 1$, so not directly applicable here. Cheng and Qian (1994) showed that when energetictrapped particle-kinetic effects are taken into account, symmetric ballooning-mirror modes are stabilized and antisymmetric modes become most unstable. The mode structure is similar to those observed by Takahashi et al. (1987) and reported here.

While the spatial structuring may well be a consequence of some sort of mirror instability, this does not explain the origin of the very high beta plasma. Very high beta values, up to 70 , have been found in the midnight sector at the end of the growth phase of a substorm and one may wonder what happens to this plasma after substorm onset. Haerendel (1999a) proposed on the basis of a first inspection of the here reported measurements that the morningside blobs may be residues of this pre-substorm high-beta plasma near Earth. This may, indeed, be the case for periods following substorm onsets in the midnight sector, but it is hard to believe that this is an explanation for the blobs found during quiet periods with no substorm preceding for many hours.

Hedgecock (1976) made the interesting proposal that the origin may be in the low-latitude boundary layer which was just discovered in those days. If we also take into consideration the occasional encounters of a reverse magnetic profile at the morningside magnetopause, as reported by Heppner et al. (1967) and also seen by Equator-S Haerendel (1999b), we have a high-beta plasma regime inside the magnetosphere on the morning side. (The evening magnetopause seems to be less prone to develop such an equilibrium with the magnetosheath plasma.) So, we may speculate that a high-beta lowlatitude boundary layer, somewhat further tailward and pervaded by closed, but highly stretched field lines is the source of the plasma blobs. The flux-tubes after having separated from this layer may move sunward under field contraction, thereby compressing the plasmas and increasing beta Erickson and Wolf (1980), and accumulating in the late night/early morning sector of the outer magnetosphere $\left(\geq 9 R_{E}\right)$. While proceeding their sunward convection, internal energy has to be expended in order to overcome the friction with the ionosphere. So, beta is slowly decreasing in this late phase of convection. During late morning hours we should thus find a less pronounced blob structure. And, indeed, this appears to be the case and will be quantified in a subsequent study.

An auroral signature of the plasma blobs has not been clearly established. At more disturbed times, in particular during substorm recovery, they may be the cause of patchy aurora. At quieter times they may manifest themselves by isolated rayed structures or have no signature altogether. The decreasing auroral occurrence rate near dawn may just indicate the decay of the blobs (magnetization) at these hours. 
The high-beta plasma blobs in the morningside magnetosphere are a new plasma regime in near-Earth space. At this time, we can only speculate about their origin, the reasons for their spatial structure, the conditions for their appearance and, last not least, their overall confinement, which typically requires the equivalent pressure of a 50-nT magnetic field, i.e. $1.0 \mathrm{nPa}$. Such pressures are not commonly found in the magnetosheath on the morning flank. Further and deeper studies are needed in order to shed light on all these topics.

Acknowledgements. The Equator-S program was supported by DARA, later DLR under grant 50 0C 94024. Our thanks belong to all teams which contributed to spacecraft development, launch, and operations and also to ESA and NASA for their contributions and support.

Topical Editor G. Chanteur thanks J-A. Sauvaud for his help in evaluating this paper.

\section{References}

Akasofu, S.-I., C.-I. Meng, and D. Kimball, Dynamics of the aurora. VI. Formation of patches and their eastward motion, J. Atmos. Terr. Phys., 28, 505-511, 1996.

Brown, W. I., J. Cahill, L. R. Davis, Acceleration of trapped Particles during a magnetic storm on April 18, 1965, J. Geophys. Res., 73, 153-164, 1968.

Cheng, C. Z., and Q. Qian, Theory of ballooning-mirror instabilities for anisotropic pressure plasmas in the magnetosphere, J. Geophys. Res., 99, 11193-11209, 1994.

Davis, T. N., and R. N. DeWitt, Twenty-four-hour observations of aurora at the southern auroral zone, J. Geophys. Res., 68, 62376241, 1968.

Erickson, G. M., and R. A. Wolf, Is steady convection possible in the Earth's magnetotail?, Geophys. Res. Lett., 7, 897-900, 1980.

Feldstein, Y. I., Some problems concerning the morphology of auroras and magnetic disturbances at high latitudes, Geomag Aeronom., 3, 183-192, 1963.

Fornacon, K.-H., H. U. Auster, E. Georgescu, W. Baumjohann, K.-H. Glaßmeier, J. Rustenbach, and M. Dunlop, The magnetic field experiment onboard Equator-S and its scientific possibilities, Ann. Geophys., 17, 1999.

Greenstadt, E. W., R. R. McPherron, R. R. Anderson, and F. L. Scarf, A storm time, Pc5 event observed in the outer magnetosphere by ISEE 1 and 2: wave properties, J. Geophys. Res., 91, 13398-13410, 1986.

Hedgecock, P. C., Giant Pc5 pulsations in the outer magnetosphere: a survey of Heos-1 data, Planet. Space Sci., 24, 921-935, 1976.
Haerendel, G., Outstanding issues in understanding the dynamics of the inner plasma sheet and ring current during storms and substorms, Adv. Space Res., 1999a.

Haerendel, G., Equator-S: the mission and first coordinated measurements with Geotail, Adv. Space Res., 1999b.

Heppner, J. P., M. Sugiura, T. L. Skillman, OGO-A magnetic field observations, J. Geophys. Res., 72, 5417-5555, 1967.

Jacobs, J. A., Geomagnetic micropulsations, Springer Verlag, Berlin Heidelberg, New York 1970

Kistler, L. M., E. Möbius, M. A. Popecki, Equator-S observations of $\mathrm{O}^{+}$beams in the dayside magnetosphere, Phys. Space Plasmas, 15, 203-207, 1998.

Lanzerotti, L. J., A. Hasegawa, and C. G. MacLennan, Drift mirror instability in the magnetosphere: Particle and field oscillations and electron heating, J. Geophys. Res., 74, 5565-5578, 1969.

Lucek, E. A., M. W. Dunlop, A. Balogh, Equator-S observations of magnetosheath mirror modes, Ann. Geophys., 18, 1999.

Möbius, E., L. M. Kistler, M. A. Popecki, The 3-D plasma distribution function analyzers with time-of-flight mass discrimination for Cluster, FAST, and Equator-S. Measure Tech Space Plasmas: Particles, 102, 243-248, 1998.

Newell, P. T., Ching-I. Meng, and K. M. Lyons, Suppression of discrete auroral by sunlight, Nature, 381, 766-767, 1996.

Nishida, A., T. Mukai, T. Yamamoto, Traversal of the nightside magnetosphere at 10-15 $R_{e}$ during northward IMF, Geophys. Res. Lett., 24, 939-942, 1997.

Oguti, T., TV observations of auroral arcs, (Physics of Auroral Arc Formation, Geophys. Monograph Ser.), A.G.U., 25, 31-41, 1981.

Price, C. P., D. W. Swift, and L.-C. Lee, Numerical simulation of nonoscillatory mirror waves at the Earth's magnetosheath, J. Geophys. Res., 91, 101-112, 1986.

Rème, H., J. M. Bosqued, J. A. Sauvaud, The CLUSTER ion spectrometry (CIS) experiment, Space Sci. Rev., 79, 303, 1997.

Southwood, D. J., A general approach to low-frequency instability in the ring current plasma, J. Geophys. Res., 81, 3340-3348, 1976.

Takahashi, K., P. R. Higbie, and D. N. Baker, Azimuthal propagation and frequency characteristic of compressional Pc 5 waves observed at geostationary orbit, J. Geophys. Res., 90, 1473-1485, 1985.

Takahashi, K., J. F. Fennell, E. Amata, and P. R. Higbie, Fieldaligned structure of storm time Pc5 wave of November 14-15, 1979, J. Geophys. Res., 92, 5857-5864, 1987.

Tsurutani, B. T., E. J. Smith, R. R. Anderson, Lion roars and nonoscillatory drift mirror waves in the magnetosheath, J. Geophys. Res., 87, 6060-6072, 1982.

Tsyganenko, N. A., Magnetospheric magnetic field model with a warped tail current sheet, Planet. Space Sci., 37, 5, 1989.

Yumoto, K., T. Saito, T. Sakurai, Local time asymmetry in the characteristics of Pc5 magnetic pulsations, Planet. Space Sci., 31, 459-471, 1983. 